\title{
EL ROL DE LA GEOGRAFÍA Y SUS HIBRIDACIONES RECIENTES FRENTE A LA CRISIS DE SUSTENTABILIDAD GLOBAL
}

\author{
Javier Osorno-Covarrubias \\ Stéphane Couturier \\ Mauricio Ricárdez \\ Laboratorio de Análisis Geo-Espacial. Instituto de Geografía. \\ Universidad Nacional Autónoma de México \\ andres@igg.unam.mx
}

\section{RESUMEN}

Presentamos una guía y un diagrama de lectura multiescalar, sistémica y «glocal» de la problemática de la sustentabilidad basada en cuatro hitos de las ciencias geográficas: 1) La formulación de las bases de la geocibernética 2) la maduración del sistema de sistemas de observación de la tierra 3) el desarrollo del concepto de limites planetarios y 4) el desarrollo de tecnologías que habilitan la participación masiva de la gente en la producción y uso de información geográfica. Se argumenta la relevancia de este enfoque para compatibilizar el sistema económico con la estabilidad ambiental.

Palabras clave: ecosfera, antropósfera, GEOSS, TIG, tierra digital, neogeografía, empoderamiento.

\section{ABSTRACT}

We present a graphical guideline of a multiscalar, systemic and «glocal» approach to the global sustainability problem, based on four hotspots of the geographical sciences: 1) The formulation of major principles of Geocybernetics, 2) the maturation of the Earth Observation System of Systems, 3) the development of the planetary limit concept, and 4)

Fecha de recepción: julio 2013

Fecha de aceptación: marzo 2015. 
the rise of technologies which enable the massive public participation in the production and use of geographic information. We argue the relevance of this viewpoint in the quest of conciliating economic welfare with environmental stability.

Keywords: ecosphere, anthroposphere, GEOSS, TIG, tierra digital, neogeography, empowerment.

\section{INTRODUCCIÓN}

La transformación a gran escala de los ecosistemas, la erosión del suelo acelerada, el cambio climático inducido por la introducción de gases con efecto invernadero, la tasa de extinción de especies, y el colapso de pesquerías son algunos de las múltiples interferencias antrópicas con registro científico sólido que actualmente comprometen la sustentabilidad de la civilización global. En este artículo revisamos cuatro hitos en la evolución reciente de las ciencias geográficas, referentes a la forma de conceptualizar, medir y operacionalizar la problemática de la sustentabilidad ambiental global.

En primer lugar, frente a la dinámica compleja de la relación sociedad-naturaleza, el enfoque merístico tradicional de las «ciencias de la tierra», exhibe limitaciones. Es cierto que es necesario cuantificar y medir los efectos ambientales con precisión, pero la urgencia de actuar invita a adoptar una estrategia de estudio que no pierde de vista el problema en su conjunto.

En la Conferencia de las Partes de las Naciones Unidas en Londres (2012), se muestran nuevamente evidencias de la amplitud y gravedad de la interferencia antrópica en el ambiente, y el alcance muy limitado de las políticas encaminadas a contener o revertir los daños. La problemática de la sustentabilidad involucra la interacción sistémica de factores ambientales, económicos, políticos y culturales, y una complejidad que genera «zonas» de incertidumbre en su comprensión y eventual modelación. Con base en estas consideraciones, este artículo empieza introduciendo la noción de 'geo-cibernética' y resalta la relevancia de sus premisas y enfoques para intentar el control de las interferencias antrópicas bajo toda clase de incertidumbres. En segundo lugar, la complejidad y el dinamismo de la civilización moderna plantean exigencias crecientes a los tomadores de decisión. La urgencia de datos y predicciones ha evolucionado más allá de la capacidad de los sistemas aislados, concebidos para un solo propósito. Estamos iniciando una nueva etapa de riesgos y oportunidades comunes en la que, la aceleración de fenómenos relacionados con el cambio global, requiere que las políticas y decisiones se sustenten en un monitoreo exhaustivo y casi-en-tiempo-real del planeta.

Por lo tanto el enfrentar con éxito estos retos parece tener como condición la existencia de políticas nacionales efectivas y una colaboración internacional a gran escala. Las acciones globales muy probablemente requieren de acuerdos políticos sustentados en la mejor información científica disponible, incluyendo datos pertinentes y oportunos acerca de los sistemas, físicos, químicos y biológicos de la tierra.

Este artículo presenta entonces dos elementos fundamentales de la infraestructura geocibernética del planeta: por un lado la integración en red de sistemas de monitoreo, predicción, alerta temprana y apoyo a la toma de decisiones en el Sistema Global de Sistemas de 
Observación de la Tierra (Global Earth Observation System of Systems-GEOSS); y por el otro el desarrollo de una infraestructura geoinformática que estblece las bases de interoperabilidad para descubrir en la red, visualizar e integrar estos recursos bajo el concepto de globos virtuales.

En tercer lugar, la dinámica de un sistema no lineal complejo puede presentar puntos de inflexión que al ser cruzados hacen que el sistema evolucione de manera brusca e irreversible a otro modo de operación o al colapso. Por esta razón, para la gestión sustentable del planeta, es crítico identificar si existen o no estos puntos de inflexión en el Sistema Tierra (ST). El siguiente hito teórico-metodológico que revisamos busca identificar y medir estos puntos de no retorno que delimitan una zona de operación segura para la humanidad. Es claro que las políticas globales de sustentabilidad deberían articularse con una altísima prioridad alrededor de estos límites.

En cuarto lugar, los efectos locales de las acciones globales y viceversa, las consecuencias globales de la acumulación de acciones locales, demandan el examen cuidadoso de la especificidad de lugares y territorios. El cuarto hito que examinamos en nuestro recorrido, es el de la evolución de tecnologías emergentes que habilitan la participación del ciudadano común en la producción, distribución y consumo de información geográfica. Se discute el potencial de estos avances para dar voz y empoderar a las organizaciones de la sociedad civil, como última línea de defensa de los bienes comunes frente a una dinámica global de expansión de la producción que «externaliza» sistemáticamente los costos sociales o ambientales.

En la discusión y conclusiones se ofrecen algunos argumentos sobre la pertinencia de los conceptos y desarrollos tecnológicos presentados, en la elaboración de una guía gráfica con visión multiescalar, sistémica y glocal, de la problemática de la sustentabilidad.

\section{LA GEOCIBERNÉTICA}

\section{II.1. El antropoceno y la necesidad imperiosa de la Geocibernética}

Sin duda uno de los grandes retos de la humanidad en el siglo XXI es el de compatibilizar el sistema económico y la protección del ambiente del que dependemos. Esta tarea de administración significa nada más y nada menos que planear y ejecutar un esquema de coevolución, aceptable y de largo plazo, entre la civilización y la naturaleza (Clark, 1989). A las bases científicas para emprender esta tarea formidable (¿imposible?) podría llamársele geocibernética, es decir, el arte de controlar adecuadamente la compleja dinámica del planeta bajo incertidumbres de toda clase.

Existen ya intervenciones en curso a escala planetaria; por un lado el proceso incesante de globalización, impulsado por la confluencia sinérgica de fuerzas de la economía, la tecnología y los estilos de vida está cambiando la faz ecológica y cultural del planeta; por otro lado existe una política ambiental global emergente que trata de reparar y prevenir los efectos colaterales más nocivos de la globalización. Una de las instancias más exitosas es la «diplomacia del ozono» para preservar el escudo de ozono en la estratosfera que protege a la biota de las radiaciones UV (Benedick, 1991). La base de evidencia científica razonablemente fundada sirvió, aunque distaba de ser perfecta, para fundamentar el protocolo de Montreal y sus adiciones que determinaron «fríamente» mecanismos para reducir y sancionar la emisión de CFCs a un nivel suficiente como para evitar una catástrofe planetaria. 
Esta ciencia difiere de la geografía, más centrada en descubrir y descifrar que modelar y predecir; difiere también de la geoingeniería, que plantea ingenuamente reparaciones directas a los «efectos colaterales» de la marcha triunfante de la civilización occidental a todo lo largo y ancho del planeta (Schellnhüber et al., 2004).

Durante toda la historia humana, con excepción de los últimos 150 años las consecuencias de la actividad humana sobre la ecosfera fueron marginales. A partir de la revolución industrial, y de manera explosivamente acentuada en los últimos 50 años emerge un nuevo modo de operación del sistema acoplado sociedad-naturaleza conocido como el modo «antropoceno» (Crutzen, 2006) que puede caracterizarse por una interferencia significativa sobre el metabolismo de la ecosfera, discernible por ejemplo en:

- El incremento antropogénico del $\mathrm{CO}_{2}$ y gases con efecto invernadero por encima de las oscilaciones naturales registradas en los últimos 850.000 años (Tripati et al., 2009).

- El volumen de agua dulce en presas y diques que es 5 veces el volumen de agua en todos los ríos (MEA, 2005a),

- La dinámica de las superficies arables en el planeta de tal magnitud que 30\% de la superficie arable del mundo es abandonada y $120,000 \mathrm{~km}^{2}$ / año son destruidos por la erosión (Pimentel et al., 1995).

- El nivel de la productividad primaria controlada por humanos que alcanza ya un $40 \%$ de la capacidad fotosintética total (MEA, 2005b)

- La tasa de extinción de especies que lleva a la desaparición de entre el 1\% y el 5\% de las especies por década; Este ritmo es entre 100 y 1000 veces superior a las tasas basal de extinciones (MEA, 2005b)

El nuevo modo de operación del Sistema Tierra, hace necesario modelar la interdependencia entre la ecosfera (E) y la antropósfera (A) considerando un nivel muy significativo de interferencia antrópica en los sistemas planetarios que tomaría la forma de estrategias deliberadas de Desarrollo y Protección del Ambiente (DyPA). Una estrategia específica M(t), constituye una secuencia cuidadosamente seleccionada de acciones de DyPA que puede consistir en un conjunto de derechos, leyes, regulaciones, incentivos, programas educativos, tratados internacionales, tecnologías e instituciones. Entonces la dinámica del complejo E-A depende sensitivamente de las estrategias seleccionadas del conjunto M. Estas estrategias definen trayectorias de coevolución T(M) generadas por diversas secuencias de decisiones (Schellnhüber y Kropp, 1998).

Para intentar siguiera ejercer este control es necesario responder a las preguntas centrales de la geocíbernética (Blackburn, 1992):

- ¿Qué tipo de mundo tenemos?

- ¿Qué tipo de mundo queremos?

- ¿Qué debemos hacer para llegar ahí?

\section{II.2. Modelar la ecosfera y la antroposfera: ¿En qué se basa la Geocibernética?}

Por lo pronto la geocibernética se ha enfocado casi exclusivamente en la ecosfera -esa compleja maquinaria bio-geo-fisico-química que sostiene la civilización humana. El moni- 

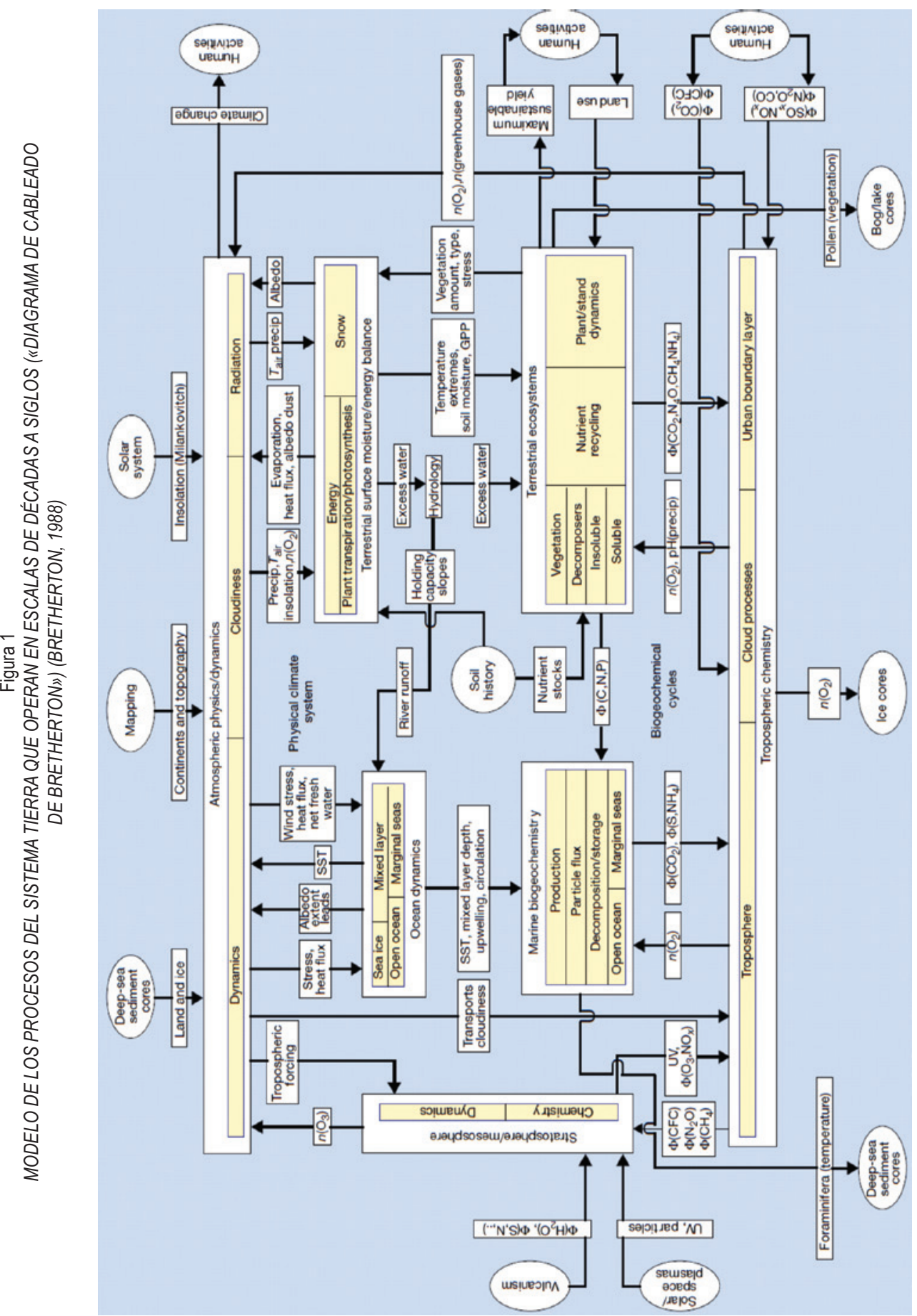
toreo, comprensión y modelación de este sistema altamente complejo, heterogéneo, adaptivo y no-lineal, es el desmedido objetivo de varios esfuerzos científicos internacionales y transdisciplinarios como el International Biosphere-Geosphere Program (IBGP); el International Human Dimensions Programme on Global Environmental Change (IHDP); el World Climate Research Programme (WCRP) y más recientemente del Global Earth Observation System of Systems (GEOSS).

Por ejemplo el ambicioso objetivo del IGBP es generar una imagen «holista» del planeta esclareciendo gradualmente los detalles del sistema tierra que Bretherton esquematizó (Figura 1: Bretherton, 1988).

La utilización de modelos de simulación es una condición sine qua non de la geocibernética (Schellnhüber, 1999). Tenemos un solo mundo y necesitamos copias virtuales, a las que sí podamos someter a toda clase de intervenciones radicales, por ejemplo suprimir las selvas tropicales, duplicar la concentración de $\mathrm{CO}_{2}$, o acidificar los océanos.

Para responder a la pregunta ¿Qué tipo de mundo queremos? podemos partir de la definición de sustentabilidad que ofrece el reporte Brundtland (WCED, 1987): «lograr las metas del presente sin comprometer la capacidad de las futuras generaciones para hacerlo». Esta máxima difusa se puede descomponer en 4 paradigmas nítidos, con principios operacionales bien definidos (tabla 1): El paradigma de estandarización prioriza el apego puntual a una normativa externa. El de optimización busca maximizar alguna función de bienestar. El de igualación antepone la equidad en el reparto de costos y beneficios. El de estabilización busca llevar al sistema hacia un estado de equilibrio aceptable.

Tabla 1

ESTRATEGIAS DE GESTIÓN DE LA CO-EVOLUCIÓN ANTROPOSFERA-ECOSFERA

\begin{tabular}{|c|c|c|c|}
\hline Símbolo & Paradigma & Meta positiva & Motivación negativa \\
\hline$P_{0}$ & Estandarización & Orden & Despotismo \\
\hline$P_{1}$ & Optimización & Prosperidad & Avaricia \\
\hline$P_{2}$ & Pesimización & Seguridad & Cobardía \\
\hline$P_{3}$ & Igualación & Equidad & Envidia \\
\hline$P_{4}$ & Estabilización & Confiabilidad & Indolencia \\
\hline
\end{tabular}

Es posible implementar combinaciones de estos paradigmas simples de DyPA bajo el espíritu de los procesos de decisión multi-objetivo (Bell et al., 1977) lo cual aportaría una mejor orientación, compatible con las preferencias reales de la sociedad global.

A pesar del progreso impresionante en el análisis de la ecosfera alcanzado en las últimas décadas, el océano de lo que resta por esclarecer parece infinito. Nuestra ignorancia respecto a las cuestiones cibernéticas puede clasificarse según las siguientes clases de incertidumbre (Peel, 2005):

- Incertidumbre cognitiva superable: que abarca los dominios de conocimiento en las que la investigación intensa termina generando información precisa sobre los procesos constitutivos y condiciones de los sistemas involucrados. 
- Incertidumbre cognitiva irreductible: todos los aspectos del sistema complejo que pueden ser determinados rigurosamente (al menos en principio) por las leyes de la naturaleza y que sin embargo no pueden ser determinados de manera nítida en un punto determinado en el tiempo (este tipo de incertidumbre es una característica distintiva de sistemas no-lineales altamente heterogéneos).

- Incertidumbre voluntaria: que abarca la indeterminación fundamental del futuro, debida a la «libertad de elección» de miles de millones de actores (individuos, corporaciones, países, instituciones multinacionales etc...).

Ante esto, ¿tendremos la osadía, o más importante aún, tenemos la más mínima posibilidad de empezar a controlar el complejísimo sistema tierra?

\section{II.3. Control difuso adaptivo: ¿Por qué la geocibernética es factible?}

Un elemento de la respuesta parece provenir de la cibernética bajo el concepto de «control difuso adaptivo». La estrategia para navegar en un laberinto de situaciones intrincadas puede expresarse de la siguiente manera: A partir de información incierta y/o incompleta adoptar una estrategia a gran escala y/o a largo plazo que es necesario reajustar continuamente de manera aproximada en base a toda clase de datos adicionales normalmente imprecisos.

Zadeh (1973) y trabajos más recientes (Kandel y Langholz, 1993) identifican tres requisitos sistémicos para que esta estrategia de control tenga posibilidades de éxito:

- La existencia de tolerancia (margen de error). Esta estrategia no funciona con sistemas quebradizos («brittel»).

- Una «reactividad» moderada considerando las velocidades con las que varía el sistema, se actualiza la información, y se implementan las medidas de respuesta.

- Una visión panorámica aproximada que garantice la orientación en el espaciotiempo a fin de evitar rutas sin salida y puntos de no retorno.

Schellnhüber y Kropp (1998) afirman que mediante la juiciosa combinación de los métodos de control que emergen de la teoría moderna de sistemas complejos y la receta de control difuso formulada arriba, es posible realizar la tarea. Este enfoque sustenta la intención de tratar de darle forma al proceso DyPA en lugar de atenerse a la intuición de que el futuro del planeta no puede ser predicho. Sin embargo, el sueño geocibernético solo puede hacerse realidad si los prerrequisitos generales enumerados arriba se operacionalizan de la siguiente manera:

- Observancia del principio de cautela (precautionary principle) que obliga a mantenerse a una distancia prudente de los límites civilizatorios y ecológicos,

- Desarrollo y cultivo de instrumentos flexibles; por ejemplo mecanismos de decisión internacional, instituciones e infraestructuras que habiliten el ajuste perpetuo (responsiveness),

- Exploración incesante de los futuros coevolutivos a través de modelos computarizados del sistema tierra (visión panorámica). 
¿Cuáles son los desarrollos instrumentales que habilitan esta visión geocibernética? La revolución de Copérnico impulsada por el telescopio, cambió la visión geocéntrica del universo y puso al planeta en el contexto astrofísico correcto. En forma análoga pero inversa, una segunda revolución copernicana es impulsada ahora por «macroscopios» que a través de la combinación de redes de sensores (remotos e in situ) y herramientas de simulación digital, permiten tomar distancia y observar una imagen holista del inventario planetario que incluye a la civilización humana, y tiene por objeto entender a la Tierra como un todo y desarrollar sobre esta base cognitiva conceptos de gestión global sustentable (Schellnhüber, 1999). Un hito relevante es la instrumentación de esta perspectiva a través de los sistemas de observación que examinamos a continuación.

\section{EL SISTEMA GLOBAL DE SISTEMAS DE OBSERVACIÓN DE LA TIERRA}

A partir de 2005, más de 70 gobiernos e instituciones, en mancuerna con más de 40 organizaciones participantes, unieron fuerza para construir un sistema global de sistemas de observación de la tierra (Global Earth Observation System of Systems-GEOSS), con el propósito de generar información de monitoreo del planeta con una operatividad y sinergia sin precedentes. Con base en un plan de trabajo a 10 años, acordado en 2005 (The Geo Secretariat, 2007), se han definido e implementado estándares técnicos de interoperabilidad, que permiten integrar las mediciones de diferentes instrumentos en conjuntos de datos temática y geográficamente exhaustivos (Rose, 2004).

El GEOSS agrupa una variedad de sensores destinados tanto para el análisis de procesos bruscos (sismos, huracanes, eventos hidrometeorológicos extremos), como de procesos graduales, relacionados con 9 áreas de beneficio societal.

\section{III.1. Características de la infraestructura}

La arquitectura con la que se implementa el GEOSS es la de un sistema integrado consistente en componentes interconectados de observación, exploración y procesamiento, intercambio y difusión de datos (Christian, 2007). Se planea que los componentes del GEOSS puedan interoperar a través de interfaces basadas en estándares internacionales abiertos (the GEO Implementation Plan Task Team: Group on Earth Observation, 2008).

\section{III.2. El alcance holístico del GEOSS}

A pesar de la masiva disponibilidad de datos de sensores es difícil alcanzar la observación holística de un fenómeno terrestre, entendiendo ésta como un conjunto de vistas de un fenómeno obtenidas por varios sensores de diferente tipo a lo largo de escalas espaciales y temporales diferentes. Un reto del GEOSS es vencer algunas fronteras, como las organizacionales y políticas, que contribuyen a esta dificultad.

A la vez de la obtención de información, se espera que analistas del clima, trabajadores en salud pública, proveedores de energía, formuladores de políticas, profesionales a nivel local y regional y expertos en temas globales estén mejor conectados. Por ejemplo, las redes de monitoreo del clima en la zona del Golfo de México y el Caribe, podrían mejorar 
la administración regional de los acuíferos, los cultivos, las zonas de pastoreo, los recursos de energía y riesgos a la salud estrechamente vinculados al clima, como brotes de dengue.

\section{III.3. La red de Sensores Web}

Uno de los componentes más relevantes del GEOSS para fines de monitoreo ambiental es la red de sensores habilitada por el conjunto de estándares Sensor Web Enablement (SWE). Este marco de interoperabilidad define un conjunto de servicios y protocolos de comunicación que permite a los desarrolladores de software conectar todo tipo de sensores, transductores y repositorios de datos de manera que sean descubribles, accesibles y utilizables a través de la Red (Botts et al., 2008). Bajo este estándar, todos los sensores reportan su posición, están conectados a la red, contienen metadatos que especifican sus recursos, ofrecen información en línea y en algunos casos, son controlables de manera remota. Bajo este paradigma, Internet está evolucionando para adquirir las capacidades de un macro instrumento sensorial activo, capaz de poner al alcance del usuario, datos de cualquier parte del mundo.

\section{III.4. El acceso masivo a datos geocientíficos a través de globos virtuales}

El concepto de Tierra Digital, o globos virtuales, prefigura el acceso de un público masivo a un volumen de datos geo-científicos creciente derivados del GEOSS. Por ejemplo, el programa de código abierto «WorldWind» de la NASA y Google Earth. Este último programa ha sido descargado más de 1,000 millones de veces y constituye un parteaguas en la popularización de la información geográfica (Google, 2014).

Se espera que algún día se reconozca como esencial esta infraestructura pública. El siguiente hito en nuestro recorrido es una innovación de carácter teórico-metodológico orientada a focalizar los esfuerzos de medición y modelación en aquellos aspectos del Sistema Tierra más propensos a sufrir transiciones bruscas e irreversibles ocasionadas por la interferencia antrópica.

\section{EL CONCEPTO DE LÍMITES PLANETARIOS}

\section{IV.1. Interferencia antrópica y transformaciones ambientales irreversibles}

A pesar de que la tierra ha sufrido muchos periodos de cambio ambiental significativo, las condiciones de la biosfera han permanecido remarcablemente estables durante los últimos 10,000 años (Schellnhüber, 1999). Este periodo de estabilidad, conocido por los geólogos como el Holoceno, ha visto a la civilización emerger, desarrollarse y medrar. Las benignas condiciones de estabilidad están ahora en riesgo. A partir de la revolución industrial emerge una nueva era, el Antropoceno, en la que las actividad humana se han convertido en la causa y motor principal del cambio ambiental.

Si bien algunos de los complejos sistemas terrestres responden de manera suave a las presiones, hay evidencia de que muchos subsistemas terrestres responden en forma no lineal y abrupta y son particularmente sensibles alrededor de valores umbral de ciertas variables críticas (Lenton et al., 2008). Al cruzar estos puntos de inflexión (PI) el subsistema «resbala» 
inexorablemente hacia un nuevo estado de equilibrio, que puede ser muy adverso y del que puede ser muy difícil regresar. Un ejemplo de ello son las trayectorias muy diferentes de deshielo y glaciación del casquete ártico: Una vez que se acumule una cantidad suficientes gases de efecto invernadero que algunos autores calculan en 450 ppm eq. $\mathrm{CO}_{2}$, iniciaría el deshielo «irreversible» del casquete de Groenlandia (Hansen et al., 2013). Para fines prácticos los daños que ocasionaría el deshielo son irreversibles, dado que el deshielo ocurre mucho más rápido que los procesos de glaciación.

El enfoque de límites planetarios busca identificar subsistemas críticos con conducta de PI. Estos umbrales pueden definirse en términos de un valor para una o más variables de control. Tal es el caso de la relación entre la concentración de $\mathrm{CO}_{2}$ en la atmósfera y el calentamiento global que se mide utilizando dos parámetros: la concentración misma y la forzante radiativa. Otros procesos, como la degradación de los servicios del ecosistema al sustituir bosques o selvas por tierras de cultivo, no tienen bordes bien definidos, pero al apartarse del valor seguro sin duda aumenta el riesgo de cruzar umbrales en otros sub-sistemas, como los mecanismos de estabilización de la maquinaria del clima (Snyder et al., 2004).

Un grupo amplio de científicos (Rockström et al., 2009) sugiere que la humanidad está cerca de los límites correspondiendo a 4 procesos: el de uso global de agua dulce, de destrucción de hábitats por cambio de uso, de acidificación de los océanos e interferencia en el ciclo global del fósforo. Por otro lado encuentran que la humanidad ya transgredió los límites de seguridad para tres procesos: el de cambio climático, la tasa de extinción de especies (entre 100 y 1000 veces la tasa basal) y la interferencia en el ciclo de nitrógeno (la incorporación antrópica de formas reactivas de nitrógeno es 4 veces superior a la incorporación natural). Para estos tres procesos, es urgente regresar a los niveles seguros para la continuidad del modo de operación holoceno. En cuanto al cambio climático se sabe con certeza que la trayectoria actual de emisión de gases de invernadero puede conducir a un incremento de $3.5^{\circ}$ a $6^{\circ}$ al final del siglo (Hansen et al, 2013). Este escenario resultaría en una compleja cascada de desastres.

Pese al consenso de la comunidad científica es muy poco lo que se ha podido hacer para frenar y revertir el crecimiento de las emisiones. En este fracaso juegan un papel decisivo las corporaciones en la industria del carbón y del petróleo, que oponen una resistencia feroz y eficiente a cualquier medida que reduzca sus ganancias espectaculares (Oreskes, 2011; Lessig, 2011, Klein, 2014). El mismo patrón se repite con las corporaciones de la minería extractiva.

\section{IV.2. Capitalismo corporativo global vs los límites del planeta}

En un estudio sobre conflictos socio ambientales en Camerún y Ecuador, Gerber (2011) presenta 58 casos. El hilo conductor en estos conflictos es el control corporativo de la tierra que desplaza a los pobladores locales y resulta en la destrucción de los usos locales del ecosistema, a medida que son reemplazados por monocultivos. La respuesta de las autoridades en la mitad de los casos fue de represión. Solo en un $20 \%$ de los casos, la movilización popular y/o los litigios judiciales, detuvieron a las concesiones.

El choque fundamental entre la economía y el ambiente se origina en dos hechos: Primero, el crecimiento demográfico; durante el siglo XX la población global se multiplicó 4 
veces y según proyecciones actualizadas puede alcanzar entre 9 mil y 11 mil millones para finales de siglo. Segundo, el metabolismo social de las economías industriales creció también en forma exponencial durante el siglo XX casi desde cualquier parámetro con él que se le mida: consumo de energía, consumo de fertilizante, utilización de dulce, intensidad de la actividad económica medida en términos del PIB real global (Steffen et al., 2011).

Esta tasa de expansión acelerada se sustenta en la intensificación en la extracción de materiales y la producción de desechos que ocurre por razones de costos y viabilidad político-militar, preferentemente en territorios periféricos del capitalismo global. En última instancia las reglas de la economía favorecen a la empresa que aumente sus márgenes de ganancia, manteniendo o reduciendo los precios a expensas de costos sociales o ambientales que no pagan en territorios periféricos. A contracorriente de esta tendencia, el movimiento del consumo responsable ha fracasado en los últimos treinta años entre otras razones porque sigue dependiendo de información escasa y del esfuerzo individual de averiguar sobre el origen y destino de productos y desechos.

¿Frente al reto de conciliar el sistema económico y los límites planetarios, qué perspectivas de empoderamiento ofrece la geografía a los actores locales? ¿Tiene algún efecto la Ciencia y Tecnología de la Información Geográfica sobre la gestión responsable de los recursos naturales? En la siguiente sección revisamos algunas de las tecnologías y prácticas emergentes que habilitan la participación de la gente en la producción, distribución y uso de información geográfica.

\section{LAS TECNOLOGÍAS DE LA INFORMACIÓN GEOGRÁFICA (TIG) EN LA PROBLEMÁTICA DE LA SUSTENTABILIDAD (TIG, PGIS, PPGIS Y VGI)}

\section{V.1. La evolución reciente de las TIG}

Tradicionalmente, por razones de costo y complejidad técnica, la producción y distribución de la información geográfica, ha sido el privilegio del Estado. Esta limitación tecnológica tiende a favorecer visiones centralistas y verticales sobre el espacio que se reflejan en las políticas públicas. En este esquema normalmente se encuentra ausente la voz de los actores locales. Dentro del cuerpo de conocimiento de la Ciencia y Tecnología de la Información Geográfica, existe un conjunto de técnicas y prácticas emergentes, que buscan el «empoderamiento»e «inclusión» de las voces y perspectivas de los actores locales.

$\mathrm{Al}$ inicio del presente milenio, el Sistema de Información Geográfica Participativo (PGIS, por sus siglas en inglés) logra acompañar un proceso de integración de saberes en un espacio local. El PGIS debe concebirse desde el inicio como un ejercicio de aprendizaje de todos los actores de manera que apuntale mecanismos equitativos de planificación, negociación y toma de decisiones (Capel, 2010). El éxito del proyecto depende de una transmisión efectiva de la información a todos los actores y de que los conocimientos se socialicen abiertamente entre los participantes.

Otro salto tecnológico-social, son las Herramientas de mapeo por Internet: Estas herramientas abren la visualización de datos espaciales a una audiencia amplísima, que accede a acervos de datos locales y remotos a través de «aplicaciones cliente» como páginas web o globos virtuales. Un ejemplo paradigmático es Google Earth que ofrece como mapa 
base un acervo planetario de imágenes de alta resolución sobrepuesta encima del modelo del terreno. El usuario puede añadir capas georeferenciadas que van desde contenidos generados por usuarios voluntarios no entrenados (Sistema de Información Geográfico Público y Participativo o PPGIS por sus siglas en inglés), hasta datos geocientíficos producto de complejos modelos de simulación (Ball, 2002; Butler, 2006). Estas herramientas, enmarcadas en la nueva geografía, habilitan la colaboración masiva de voluntarios (Información Geográfica generada Voluntariamente, o VGI, por sus siglas en inglés) en la producción (crowdsourcing) y distribución (crowdcasting) de contenidos geográficos (Elwood et al., 2013; Goodchild y Michael, 2007; Hudson-Smith et al., 2009; Sun et al., 2009).

\section{V.2. Integración de conocimientos a escala local}

Varios autores (Anderson et al., 2009; Capel, 2010; Elwood, 2006; Stewart et al., 2008) reportan en la literatura casos en los que se utilizan las TIG en litigios entre comunidades ancestrales y gobiernos, relacionados con el uso de recursos naturales, a través de un proceso de SIG Participativo (SIGP). Un ejemplo característico es el litigio de la comunidad Ogiek con el gobierno de Kenia por su desalojo del bosque Mau Oriental y la reubicación en esa zona de agricultores de otras regiones. Esta comunidad participó en un proyecto en el que se emplearon fotografías aéreas y técnicas de cartografía participativa para elaborar un mapa de los territorios ancestrales (Ehrensperger et al., 2007). Este tipo de técnicas se ha utilizado para la integración de diferentes conocimientos en un solo instrumento que se emplea en procesos de negociación y planificación (Bell et al., 1977). También permite formular de manera eficaz las reclamaciones territoriales de la comunidad y apuntala la inclusión de sus perspectivas en los procesos de planeación (ej. Hall et al., 2010; Sieber, 2000).

Los participantes en el proceso de negociación son inducidos a proporcionar información más precisa y transparente. La expresión de la perspectiva de la comunidad resulta entonces más eficaz frente a las autoridades (Ghose, 2007), que las expresiones que se valen solamente de la narración o el bosquejo de mapas. Contribuye por lo tanto a una mayor certidumbre de los acuerdos generados en la planificación y la toma de decisiones.

\section{V.3. La producción colaborativa de información geográfica}

La revolución informática asociada al salto de la Web 1.0 a la 2.0 (O’Reilly, 2004) abrió el paso a la participación del público en la producción de contenidos e instrumentó la proliferación de páginas, blogs, wikis, redes sociales, y otros mecanismos de difusión centrados en el usuario y generados de manera distribuida y colaborativa. El correlato geográfico de este proceso se ha denominado la GeoWeb 2.0.

$\mathrm{Al}$ final de la primera década emergen nuevas formas de creación y socialización de contenidos geográficos, conocidas colectivamente como VGI. Estas nuevas formas de construir acervos espaciales, habilitadas por la proliferación de dispositivos móviles conectados a internet, presenta modalidades inéditas: Se trata de una colaboración descentralizada, espontanea, masiva, que contribuye a darle color y significado a los espacios locales. Una de las modalidades de VGI es la ciencia ciudadana y en particular Colaborative Sensing (ejemplo mapas de ruido, mapas de contaminación del aire, mapas de humor («happiness maps»)). 
Destacan los acervos geográficos planetarios libres como la Wikimapia (Goodchild et al., 2010) y OpenStreetMap (Haklay, 2010) también la emergencia espontanea de sitios de respuesta inmediata en situaciones de crisis.

\section{V.4. ¿La nueva geografía propiciada por una democratización de la tecnología?}

La popularización de la tecnología geoespacial entró en fase acelerada impulsada por el abaratamiento de dispositivos de cómputo móvil permanentemente conectados a la red. La divisa de esta transformación puede condensarse en la frase «poner la información al alcance de todos, desde cualquier parte y en cualquier momento» (anyone, anyware, anytime). ¿Significa esto una democratización de la tecnología? ¿Empodera al ciudadano promedio?

Los indicios son mixtos y el balance a futuro es de difícil pronóstico. Si por democratización se entiende popularización, para finales de 2014, el uso de teléfonos inteligentes habrá ascendido a 1,700 millones de usuarios (eMarketer, 2014). Si por democratización entendemos el respeto a la libertad de expresión y la privacidad, el panorama es distinto. Cada uno de estos usuarios, lleva una antena en el bolsillo que difunde su posición a las empresas que proveen el servicio. El uso que dan los proveedores de servicio a este registro espacio-temporal, personalizado y detallado es opaco y pone en riesgo la privacidad del usuario. Un episodio ilustrativo de la falta de respeto a la privacidad quedo al descubierto cuando la revista Wired, publicó el hallazgo de dos hackers que descubren en cada iPhone con el sistema operativo iOS 4, un archivo con el registro espacio-temporal meticuloso que se estuvo grabando durante 10 meses, a partir del despliegue de esta versión del sistema operativo, sin que nadie lo supiera (Tene y Polonetsky, 2013). El asunto generó escándalo cuando se publicó, pero no ha modificado las débiles regulaciones de protección a la privacidad vigentes (Andrejevic, 2013). El monitoreo de ubicación persistente implica un control no en un sentido físico sino en un sentido Orweliano en el que la vigilancia permanente y estrecha juega un papel en el miedo de un individuo de ser pescado «fuera de lugar». Las revelaciones de Edward Snowden dan cuanta del espionaje global y sistemático sobre las comunicaciones privadas que ejerce la NSA de Estados Unidos (Greenwald, 2013). Ni qué decir de países como China o Rusia, en los que hay un control y censura abiertos del correo electrónico y las redes sociales.

En sentido inverso cabe mencionar tres instancias de aplicación de TIGs que apuntalan a una vigilancia colaborativa del uso sustentable de los recursos naturales globales: 1) Global Fishing Watch es producto de una colaboración de las ONGs Sky Truth y Oceana, y del corporativo Google para construir una herramienta Web que permite mostrar todas las actividades rastreables de pesca oceánica en el planeta (Global Fish Watch, 2014). 2) La ONG Apalachian Voices utiliza Google Earth para enterar a la gente de la escala de destrucción que produce la minería de carbón a cielo abierto (Appalachian Voices, 2014). 3) El proyecto «Atlas de Justicia Ambiental» de la ONG «Environmental Justice Organizations, Liabilities and Trade» (Ejolt, 2013) pone a disposición del público una plataforma en línea, práctica e intuitiva, que permite visualizar, filtrar y seleccionar conflictos ambientales graves a nivel global. A través de estas plataformas se espera reducir la ceguera del consumidor y sugerir políticas de gestión y cambios de consumo que conduzcan a sistemas de producción ecológica y socialmente aceptables. 


\section{DISCUSIÓN}

En 1997 la tercera conferencia de las partes de la Convención sobre Cambio Climático de las Naciones Unidas en Kyoto fue un punto de inflexión histórico que apuntaba al inicio de la era geocibernética. Fue la primera vez que las Naciones Unidas buscaron ejercer control consensuado sobre un asunto a escala global, pese a que implicaba una afectación negativa a los intereses de corto plazo de miles de millones de personas. 17 años después la concentración atmosférica de $\mathrm{CO}_{2}$ pasó de 280 a 400 ppm, el valor más alto por lo menos en los últimos 850,000 años (Tripati et al., 2009). Las emisiones lejos de desacelerar crecen a una tasa record del $3 \%$.

Este resultado contrasta con el de otra intervención, esta sí exitosa, que permitió preservar, el escudo de ozono en la estratosfera que protege la biota de las radiaciones ultravioleta. ¿Cuál es la diferencia entre estos dos resultados? El nivel de influencia sobre los resortes del poder de las industrias involucradas: en el primer caso era solamente una corporación, la «Dupont» que tenía la patente para producir freón como refrigerante y propelente (Shellnhüber, 1999). En el segundo caso es la industria completa de energía fósil (petróleo, gas y carbón) con recursos de sobra para gastar en think thanks, campañas de presión en los medios de difusión, donaciones en campañas electorales y «lobbies» profesionales que influyen sobre los procesos legislativos (Lessig, 2011, Diesendorf, 2014, Elsner, 2014).

Mientras que las fronteras nacionales se han vuelto casi irrelevantes para los actores económicos globales, los derechos humanos y las regulaciones de protección del ambiente, están confinadas y dominadas por una miríada de fronteras. Como prerrequisito se perfila la necesidad de transformar los mecanismos de control simbólico de las Naciones Unidas por instituciones supranacionales poderosas y eficaces (Stangu, 2010).

$\mathrm{Al}$ otro extremo, dada la opacidad e impunidad con la que opera la «externalización» corporativa de costos, los ejemplos de apropiación de las TIG en beneficio de la comunidad deben multiplicarse. Hacklay (2013) nos recuerda que las tecnologías no son inocentes, y que para cubrir la promesa de empoderamiento comunitario que se deriva de su uso eficaz, es necesario hackearlas. El software espacial de código libre y abierto, y los acervos espaciales libres abren un espacio fértil a la colaboración entre la academia y la sociedad civil.

\section{CONCLUSIONES}

No cabe duda que vivimos tiempos de cambio acelerado. La problemática relación sociedad-naturaleza amenaza con empujar a la ecosfera a situaciones de cambio abrupto y desastroso para amplios sectores de la humanidad. ¿Vamos hacia un colapso ambiental global? ¿Podemos evitarlo?

En la primera sección se examina la pertinencia de la modelación geocibernética que deriva 3 requisitos para el control difuso adaptivo del sistema acoplado ecosfera-antroposfera: 1) Mantenernos a una distancia prudente de los límites civilizatorios y ecológicos; 2) Desarrollar y cultivar mecanismos flexibles a diferentes escalas, instituciones e infraestructuras que habiliten el ajuste perpetuo; 3) Explorar futuros co-evolutivos a través de modelos computarizados que nos ofrecen una visión panorámica de donde estamos y hacia dónde vamos. 
La formulación del concepto de límites planetarios establece bordes difusos que no debemos transgredir. Ahora sabemos que ya traspasamos tres de estos bordes y que debemos regresar cuanto antes a los límites seguros para evitar daños inadmisibles. Pese a la claridad de las advertencias de la comunidad científica, en estos tres frentes es muy poco lo que se ha hecho. Hay una acumulación creciente de indicios que señalan la resistencia de las corporaciones globales y su peso excesivo sobre los resortes del poder a diferentes escalas, como un factor decisivo en esta trayectoria riesgosa para todos.

La visión de Goodchild y Michael (2007) en la que 6 mil millones de personas, empoderadas por tecnologías de comunicación y computo móvil que registran y comunican su percepción de los hechos, tiene visos de cumplirse antes del 2030 (Diamandis y Kotler, 2012). Es probable que las 3,000 millones de voces nuevas, la mayoría en espacios periféricos del capitalismo, contribuyan a visibilizar los destrozos e injusticias a nivel del piso, y evidenciar el «progreso» desigual y dispendioso. Esto permitiría cerrar la pinza de control geocibernético reflexivo, desde la escala local a la escala planetaria.

A manera de síntesis, proponemos una representación (figura 2) de un acercamiento multiescalar, sistémico y glocal de la problemática de la sustentabilidad desde las Ciencias Geográficas:

Figura 2

DIAGRAMA DE LECTURA MULTIESCALAR, SISTÉMICA Y GLOCAL DE LA PROBLEMÁTICA DE LA SUSTENTABILIDAD CON RECURSOS DE LAS CIENCIAS GEOGRÁFICAS

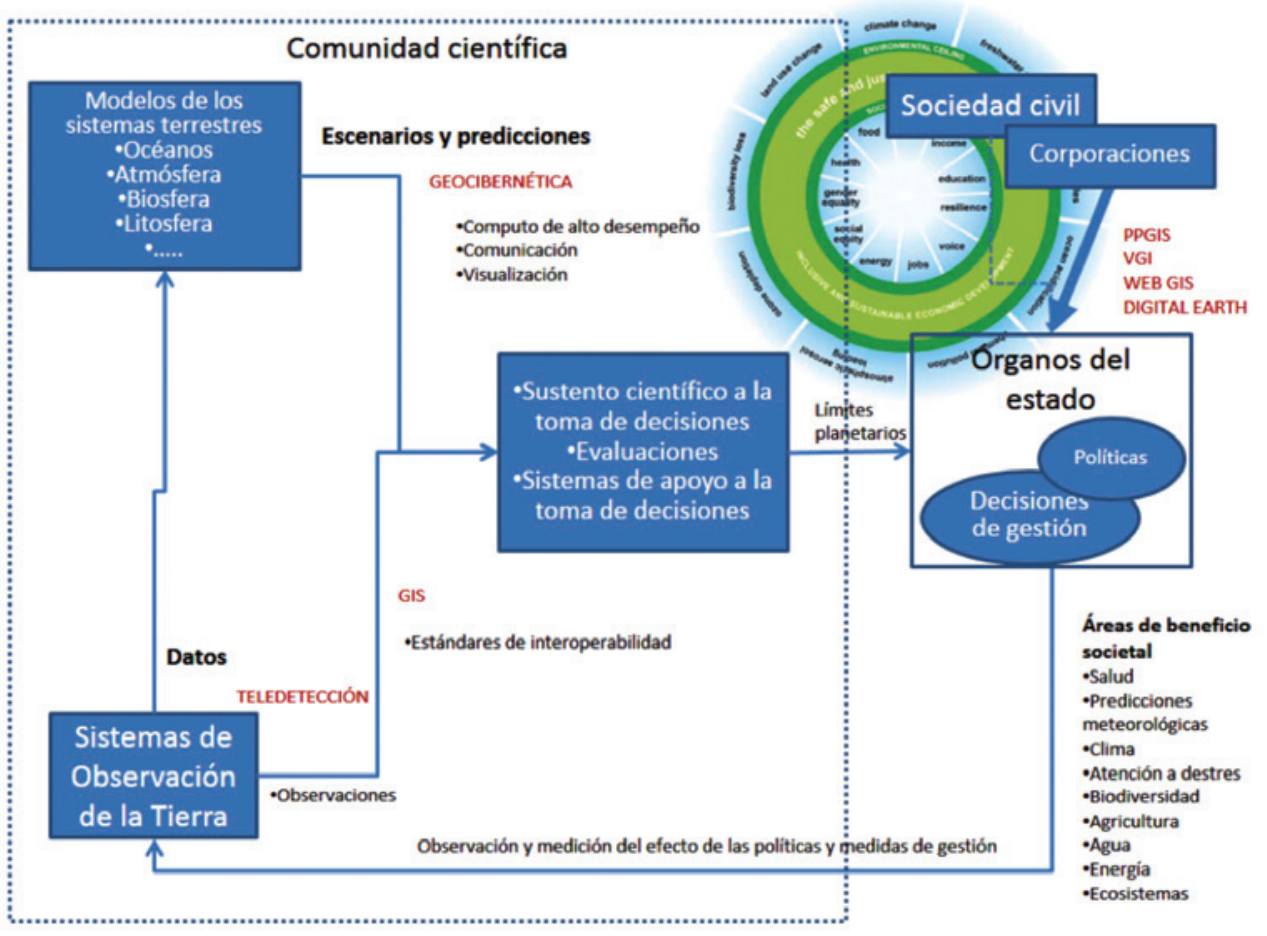


En un primer tiempo, los Modelos de los Sistemas Terrestres (1) describen el comportamiento de la compleja maquinaria biogeoquímica. Estos modelos están sujetos a un perfeccionamiento recursivo basado en el contraste entre observaciones de la red de sensores (2) de los Sistemas de Observación de la Tierra (GEOSS). En función de los límites planetarios identificados por estos modelos, la comunidad científica provee el Sustento Científico a la toma de decisiones (3) a la vez que el mismo GEOSS permite observar el efecto de estas decisiones (4). Sin embargo, este escenario idealizado del rol de la ciencia en la formulación y operación de políticas se ve obstaculizado sistemáticamente por el poder decisivo de las corporaciones globales (5). De aquí la necesidad de «hackear» las Tecnologías de la Información Geográfica (PPGIS, VGI) para resistir y revertir la externalización de costos. En este proceso es fundamental el rol de la Sociedad Civil (6), apoyada por la Comunidad Científica (redes de observadores), para definir y defender, desde cada territorio, un piso social de bienestar y equidad.

En última instancia la crisis ambiental actual nos ofrece una valiosa lección. Todos vamos en el mismo barco. Nos conviene actuar en forma equitativa y solidaria o corremos el riesgo de hundirnos.

\section{AGRADECIMIENTOS}

Varios proyectos de investigación permitieron la reflexión presentada en este artículo y la formulación aquí propuesta. En un primer tiempo, una búsqueda exhaustiva de antecedentes se realizó en el ámbito de dos proyectos financiados por la DGAPA de la Universidad UNAM: El proyecto IACOD número IC300111, titulado «Contribución geocibernética a la observación territorial de amenazas y riesgos», y el proyecto PAPIIT número RA300113, titulado «Confiabilidad cartográfica de procesos territoriales: un acercamiento con lógica difusa para incorporar múltiples actores sociales».

Más recientemente, la formulación conceptual se logró obtener en el ámbito del proyecto número IA300515, igualmente financiado por la DGAPA - UNAM y titulado «Una caracterización territorial multiescalar sobre la contribución de la sociedad mexicana al cambio ambiental global». Agradecemos en consecuencia a la instancia financiadora pública DGAPA-UNAM que contribuyó a la realización de esta investigación.

\section{BIBLIOGRAFÍA}

ANDERSON, C., BEAZLEY, K. et al. (2009): «Lessons for PPGIS from the application of a decision-support tool in the Nova Forest Alliance of Nova Scotia, Canada». Journal of Environmental Management 90(6): 2081-2089.

ANDREJEVIC, M. (2013): Estranged free labor. In: Digital Labor: the Internet as Playground and Factory. Springer, New York, 149-164.

APPALACHIAN VOICES (2014): Protecting the Central \& Southern Appalachian Mountain Region. Página Internet accesible en: http://www.appalachianvoices.org/

BALL, J. (2002): «Towards a methodology for mapping 'regions for sustainability' using PPGIS». Progress in Planning 58: 81-140.

BELL, D.E., KEENEY, R.L., RAIFFA, H. (1977): Conflicting objectives in decisions. Wiley, Londres. 
BENEDICK R.E. (1991): Ozone diplomacy: new directions in safeguarding the planet. Harvard University Press, Cambridge.

BLACKBURN, C. (1992): Summary, conclusions, and recommendations. Global change and the human prospect: issues in population, science, technology and equity. Sigma Xi, The Scientific Research Society.

BOTTS, M., PERCIVALL, G., REED, C. y DAVIDSON, J. (2008): «OGC Sensor Web Enablement: Overview and High Level Architecture». GSN 2006, LNCS, Volume 4540. pp. 175-190.

BUTLER, D. (2006). «Virtual globes: The web-wide world.» Nature, 439(7078): 776-778.

BRETHERTON, F. (1988): Earth System Sciences: A Closer View. Earth System Sciences Committee, NASA.

CAPEL, H. (2010): «Networked Geography at the beginning of the Third Millennium: for a solidary and collaborative science». Scripta Nova.Revista Electronica de Geografia Y Ciencias Sociales 14(313).

CHRISTIAN, E. (2007): GEOSS Architecture principles and the GEOSS clearinghouse. The Full Picture: 106-108.

CLARK W.C. (1989): «Managing planet Earth». Scientific American 261(9): 46-54.

CRUTZEN, P. J. (2006). The «anthropocene». Earth System Science in the Anthropocene, Springer: $13-18$.

DIAMANDIS, P.H., y KOTLER, S. (2012). Abundance: The future is better than you think. Simon and Schuster, 232p.

DIESENDORF, M. (2014): «Corporate efforts to impede renewable energy.» Chain Reac$\operatorname{tion}(121): 38-40$.

EHRENSPERGER, A., WYMANN VON DACH, S. et al. (2007): «Tecnologías de la Información Geográfica para el Manejo de los Recursos Renovables». InfoResources Focus. InfoResources, Centre for Development and Environment. 07: 16.

EJOLT (2013): «Environmental Justice Organisations, Liabilities and Trade», página internet, accesible en: http://www.ejolt.org/

ELWOOD, S. (2006): «Negotiating knowledge production: The everyday inclusions, exclusions, and contradictions of participatory GIS research». Professional Geographer 58(2): 197-208.

ELWOOD, S., GOODCHILD, M.F., y SUI, D. (2013): Prospects for VGI research and the emerging fourth paradigm. In Crowdsourcing Geographic Knowledge, Springer: 361375.

Environmental Support Solutions (2009): Global Warming and Ozone Depletion. Disponible en http://www.ess-home.com/news/global-warming/ozone-depletion.asp.

ELSNER, G. (2014): «Using Public Relations to Defy Science.» Journal of Mass Media Ethics 29(2): 131-134.

eMARKETER (2014): Sitio Web de la empresa eMarketer. Acceso en: http://www.emarketer.com/

GERBER, J.F. (2011): Conflicts over industrial tree plantations in the South: Who, how and why?. Global Environmental Change, 21(1), 165-176.

GHOSE, R. (2007): «Politics of scale and networks of association in public participation GIS». Environment and Planning A, 39(8): 1961-1980. 
GLOBAL FISHING WATCH (2014): A Technology Initiative to Illuminate Global Fishing Activity, Página Web accesible en: http://globalfishingwatch.org/

GOODCHILD, M.F. y MICHAEL F. «Citizens as sensors: the world of volunteered geography.» GeoJournal 69.4 (2007): 211-221.

GOODCHILD, M.F. y GLENNON, J.A. (2010): «Crowdsourcing geographic information for disaster response: a research frontier». International Journal of Digital Earth 3(3): 231-241.

GRAHAM, M. (2010): «Neogeography and the palimpsests of place: web 2.0 and the construction of a virtual earth». Tijdschrift Voor Economische En Sociale Geografie 101(4): 422-436.

GREENWALD, G. (2013): «XKeyscore: NSA tool collects' nearly everything a user does on the internet'.» The Guardian.

GOOGLE (2014): «Google Earth downloaded more than one billion times.» Google Maps. Retrieved 3/12/2014, 2014, from http://google-latlong.blogspot.mx/2011/10/googleearth-downloaded-more-than-one.html

GROUP ON EARTH OBSERVATIONS (2008): GEO Information Sheets, GEO: 1-30.

HAKLAY, M. (2010). «How good is volunteered geographical information? A comparative study of OpenStreetMap and Ordnance Survey datasets.» Environment and planning. B, Planning \& design 37(4): 682.

HAKLAY, M. (2013): «Neogeography and the delusion of democratisation.» Environment and Planning A 45(1): 55-69.

HANSEN, J., KHARECHA, P., SATO, M., MASSON-DELMOTTE, V., ACKERMAN, F., BEERLING, D.J. y ZACHOS, J.C. (2013): Assessing «Dangerous Climate Change»: required reduction of carbon emissions to protect young people, future generations and nature. PloS one, 8(12), e81648.

HALL, G. B., CHIPENIUK, R. et al. (2010): «Community-based production of geographic information using open source software and Web 2.0». International Journal of Geographical Information Science 24(5): 761-781.

HUDSON-SMITH, A., BATTY, M. et al. (2009): «Mapping for the Masses Accessing Web 2.0 Through Crowdsourcing» Social Science Computer Review 27(4): 524-538.

KANDEL, A. y LANGHOLZ, G. (1993). Fuzzy control systems. Crc press.

KLEIN, N. (2011). «Capitalism vs. the Climate.» The Nation 28: 11-21.

LENTON, T.M., HELD, H., KRIEGLER, E., HALL, J.W., LUCHT, W., RAHMSTORF, S., \& SCHELLNHUBER, H.J. (2008). Tipping elements in the Earth's climate system. Proceedings of the National Academy of Sciences, 105(6), 1786-1793.

LESSIG, L. (2011): Republic, Lost: How Money Corrupts Congress--and a Plan to Stop It, Hachette Digital, Inc.

MEA (Millenium Ecosystem Assessment) (2005a): Ecosystems and human well-being: synthesis. Island Press, Washington D.C., EEUU.

MEA (Millenium Ecosystem Assessment (2005b): Ecosystems and human well-being: biodiversity synthesis. Island Press, Washington D.C., EEUU.

O'REILLY, T. (2004): «Radar, Insight, Analysis, and Research about Emerging Technologies», revista en línea. Acceso en: http://radar.oreilly.com/archives/2005/10/web_20 _compact_definition.html 
ORESKES, N. y CONWAY, E.M. (2010): Merchants of Doubt: How a Handful of Scientists Obscured the Truth on Issues from Tobacco Smoke to Global Warming, Blumbsbury Press.

PEEL, J. (2005): The precautionary principle in practice: Environmental decision-making and scientific uncertainty. Federation Press.

PIMENTEL, D., HARVEY, C., RESOSUDARMO, P., SINCLAIR, K., KURZ, D. y BLAIR, R. (1995): «Environmental and economic costs of soil erosion and conservation benefits». Science, 267 (5201), 1117-1123.

REYES, C., TAYLOR, D.R.F. et al. (2006): «Geo-cybernetics: A New Avenue of Research in Geomatics?» Cartographica 41(1): 15.

ROCKSTRÖM, J., STEFFEN, W. et al. (2009): «A safe operating space for humanity». Nature 461(7263): 472-475.

ROSE, L. C. (2004): Geospatial Portal Reference Architecture, Open Geospatial Consortium.

SCHELLNHÜBER, H., CRUTZEN, P. et al., Eds. (2004): Earth System Analysis for Sustainability.

SCHELLNHÜBER, H.J. (1999): «Earth system analysis and the second Copernican revolution». Nature 402: C19-C23.

SCHELLNHÜBER, H. y KROPP, J. (1998): «Geocybernetics: Controlling a Complex Dynamical System Under Uncertainty». Naturwiessenshaften (85): 14.

SIEBER, R.E. (2000): «Conforming (to) the opposition: the social construction of geographical information systems in social movements». International Journal of Geographical Information Science 14(8): 775-793.

SNYDER, P.K., DELIRE, C., y FOLEY, J.A. (2004): «Evaluating the influence of different vegetation biomes on the global climate». Climate Dynamics, 23(3-4), 279-302.

STANGU, I. (2010): «GeoCybernetics -a 21 century cybernetic approach to sustainable development and environmental protection». FIG Congress 2010. Facing the Challenges - Building the Capacity, Sydney, Australia.

STEFFEN, W., ROCKSTRÖM, J. Y COSTANZA, R. (2011): «How defining planetary boundaries can transform our approach to growth». Solutions, 2(3).

STEWART, E. J., D. JACOBSON, et al. (2008): «Public participation geographic information systems (PPGIS): challenges of implementation in Churchill, Manitoba». Canadian Geographer-Geographe Canadien 52(3): 351-366.

SUN, M.T.W., TSAI, Y.T. et al. (2009): «Public participation and the concept of space in environmental governance: an application of PPGIS». Public Administration and Development 29(3): 250-261.

TENE, O. y POLONETSKY, J. (2013): A theory of creepy: technology, privacy and shifting social norms. Yale Journal of Law \& Technology, 59, 16.

THE GEO SECRETARIAT (2007): The Full Picture. Earth Observations for Sustainable Growth and Development. Switzerland, Tudor Rose.

TRIPATI, A. K. et al. (2009): «Coupling of $\mathrm{CO}_{2}$ and ice sheet stability over major climate transitions of the last 20 million years». Science 326(5958): 1394-1397.

TZIPERMAN, E., SCHER, H., ZEBIAK, S.E. y CANE, M.A. (1997): «Controlling spatiotemporal chaos in a realistic El Niño prediction model». Physics Review Letter 79: 1034-1037. 
WCED (1987): Our Common Future (The Brundtland Report), United Nations World Commission on Environment and Development.

WRIGHT, R.T. y NEBEL, B.J. (2002): Environmental Science: Toward a Sustainable Future, Prentice Hall.

ZADEH, L.A., (1973): «Outline of a new approach to the analysis of complex systems and decision processes», IEEE Transactions on Systems, Man, and Cybernetics, Vol. SMC-3 (1), 28-45. 